\title{
Inverse reconstruction technique based on time-dependent Petschek-type reconnection model: first application to THEMIS magnetotail observations
}

\author{
V. Ivanova ${ }^{1}$, J. Liu ${ }^{2}$, S. Kiehas ${ }^{1}$, V. Semenov ${ }^{3}$, and H. Biernat ${ }^{1}$ \\ ${ }^{1}$ Space Research Institute, Austrian Academy of Sciences, 8042 Graz, Austria \\ ${ }^{2}$ IGPP/ESS, University of California, Los Angeles, CA 90095-1567, USA \\ ${ }^{3}$ Institute of Physics, St-Petersburg State University, 198504 St-Petersburg, Russia \\ Received: 20 August 2009 - Accepted: 26 November 2009 - Published: 2 December 2009
}

\begin{abstract}
We apply the inverse reconstruction technique based on the two-dimensional time-dependent Petschek-type reconnection model to a dual bipolar magnetic structure observed by THEMIS B probe in the Earth's magnetotail during a substorm on 22 February 2008 around 04:35 UT. The technique exploits the recorded bipolar magnetic field variation as an input and provides the reconnection electric field and the location of the X-line as an output. As a result of the technique application, we get (1) the electric field, reaching $\sim 1.1 \mathrm{mV} / \mathrm{m}$ at the maximum and consisting of two successive pulses with total duration of $\sim 6 \mathrm{~min}$, and (2) the approximate X-line position located in the magnetotail between 18 and $20 R_{E}$.
\end{abstract}

Keywords. Space plasma physics (Magnetic reconnection)

\section{Introduction}

An attempt to get a remote estimate of the electric field along the X-line in the Earth's magnetotail was made by Richardson and Cowley $(1985,1987)$. Using the dispersion of ion bursts and the expansion speed of the boundary layer, measured by ISEE3 spacecraft, they deduced the electric field along the near-Earth reconnection line. However, since the ISEE3 spacecraft was located far away from the proposed Xline location, the estimates obtained by them were not conclusive.

Recently, a novel remote-sensing technique was introduced (Semenov et al., 2005), which allows to reconstruct the time-varying reconnection rate and the $\mathrm{X}$-line location

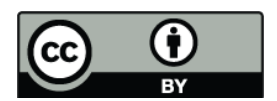

Correspondence to: V. Ivanova

(biglion@inbox.ru) from single spacecraft magnetic data. The technique was successfully applied to both: isolated reconnection events in the magnetotail (Ivanova et al., 2007), like NFTEs (Nightside Flux Transfer Events) or TCRs (Travelling Compression Regions), and composite reconnection events consisting of several successive NFTE/TCR-signatures (Ivanova et al., 2008). Under the term "successfully" we mean that reasonable physical values were obtained for the reconnection rate and the $\mathrm{X}$-line location.

To exploit the reconstruction tool, one needs (1) a record of reconnection-associated bipolar magnetic variations, obtained in the tail lobes or, at least, at the plasma sheet periphery/PSBL (Plasma Sheet Boundary Layer), and (2) an estimate of the average propagation velocity of these bipolar structures along the Sun-Earth direction. All numerical calculations are carried out in the normalized form and knowledge of the propagation velocity is needed to convert reconstruction results to dimensional values.

It is important to note that the technique implies a homogeneous background in the tail. In particular, the propagation velocity of magnetic structures is assumed to be constant everywhere between the X-line and a point of the spacecraft observation. In reality, of course, there is no homogeneity along the Sun-Earth direction and the velocity does not stay constant. This is the first factor, which reduces the accuracy of the method. The second one is the problem of reliable determination of the average propagation velocity. Essential uncertainties in estimating this quantity often occur, thus decreasing validity of the reconstruction results. As it was stated above, the technique itself require single spacecraft measurements. However, use of multiple spacecraft is necessary because it gives an opportunity to estimate the magnetic structure velocity. In case of CLUSTER the propagation velocity of NFTE/TCR-structures can be found by means of

Published by Copernicus Publications on behalf of the European Geosciences Union. 


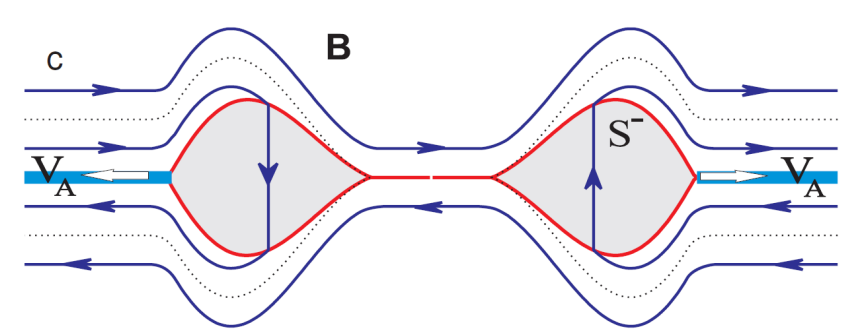

Fig. 1. Time-dependent Petschek-type reconnection model: Current sheet separating two plasma domains with opposite magnetic fields $B$ decays into a system of MHD discontinuities and shocks $S$, which form two outflow bulges propagating in opposite directions with the Alfvén speed $V_{A}$. The dotted line is a separatrix between the reconnected magnetic flux and the non-reconnected one. The shaddowed area represents outflow bulges themselves.

multi-point timing (though, strictly speaking, this approach gives the local velocity at the point of observation instead of the average one.) For THEMIS mission a necessary estimate of the average propagation velocity may be obtained by tracing a reconnection-associated structure through the sequence of THEMIS spacecraft.

Up to now the technique was applied to CLUSTER observations only. In this paper we present first application of our method to magnetotail data obtained by THEMIS. We investigate a tailward propagating composite magnetic structure consisting of two bipolar $B_{z}$-variations (where $z$ is the direction normal to the current sheet), which was detected by THEMIS B probe during a substorm on 22 February 2008 around 04:35 UT. Our motivation to use THEMIS data was based on a hope to get a reliable estimated of really the average propagation velocity (instead of the local one).

\section{Inverse reconstruction technique based on time- dependent Petschek-type reconnection}

For convenience of the reader a brief description of the reconstruction tool is given in this section. Further details can be found in the papers by Semenov et al. (2005), where the first variant of the reconstruction technique is presented (the limit of incompressible plasma), and by Ivanova et al. (2007), where extension for compressible plasma is given.

The reconstruction tool was developed on the basis of 2-D time-dependent Petschek-type reconnection model (Semenov et al., 1983, 2004; Heyn and Semenov, 1996), which generalizes the classic Petschek mechanism for an unsteady regime. The unsteady solution allows to simulate different reconnection regimes (impulsive, quasi-stationary, intermittent) and, thus, to investigate dynamics of the reconnection process depending on a variable reconnection rate. It also describes the current sheet state after reconnection has ceased.
In the frame of this model the reconnection rate (the electric field at the $\mathrm{X}$-line) is prescribed a priori as an arbitrary function of time restricted by the causality: $E(t) \equiv 0$ for $t \leq 0$. Local appearance of the electric field leads to a decay of the current sheet into a system of MHD discontinuities and shocks (Biernat et al., 1987; Heyn et al., 1988), which form two outflow bulges containing accelerated plasma (Fig. 1). Once reconnection ceases (i.e. the electric field at the X-line drops to zero), the outflow bulges detach themselves from the reconnection site and move in opposite directions along the current sheet.

The model is analytical and is predicated on a number of simplifying assumptions: (i) plasmas and magnetic fields in reconnecting domains are homogeneous; (ii) the initial current sheet contains no normal component and is thin enough to be approximated by a tangential discontinuity; and (iii) the reconnection electric field is much less than the electric field calculated from the background magnetic field and the Alfvén velocity $E \ll E_{A}=v_{A} B_{0} / c$ (Petschek, 1964).

Perturbations caused by the moving outflow bulges in the surrounding medium are found from the set of compressible ideal MHD equations linearised with respect to the constant background. External perturbations (observed outside the bulges) can be written in the form of a convolution integral:

$B_{z}(t, x, z)=\int_{0}^{t} d \tau K_{z}(\tau, x, z) E(t-\tau)$.

Here $E$ is the reconnection electric field, $x$ and $z$ are the observational coordinates along and normal to the current sheet, counted from the reconnection site (X-line).

The kernel of convolution $K_{z}(\tau, x, z)$ contains information on: (1) medium parameters; (2) waves and discontinuities launched by reconnection; and (3) position of observation with respect to $X$-line. Physically, the kernel characterizes the response of the medium to an elementary, delta-shaped pulse of reconnection (Penz et al., 2006).

External perturbations are caused by bending of magnetic field lines around the outflow bulge and, since the shape of the bulge depends on the time profile of $E(t)$, they, naturally, reflect all changes in the reconnection rate (see Eq. 1). For an intermittent electric field consisting of two successive pulses the model predicts a dual bipolar $B_{z}$-variation and a dual $B_{x}$ compression (Fig. 2).

Qualitative agreement between model predictions and spacecraft observations gave rise to an idea of inverting the problem. The inverse solution gives the reconnection electric field if magnetic perturbations are known (whereas the direct solution defines perturbations for the specified electric field). Indeed, if the magnetic variation $B_{z}(t)$ at some observational point $(x, z)$ is known (from spacecraft measurements), the relation (1) can be seen as an integral equation for the unknown electric field $E(t)$. Employing a standard method for solving integral equations of the convolution type, namely, 

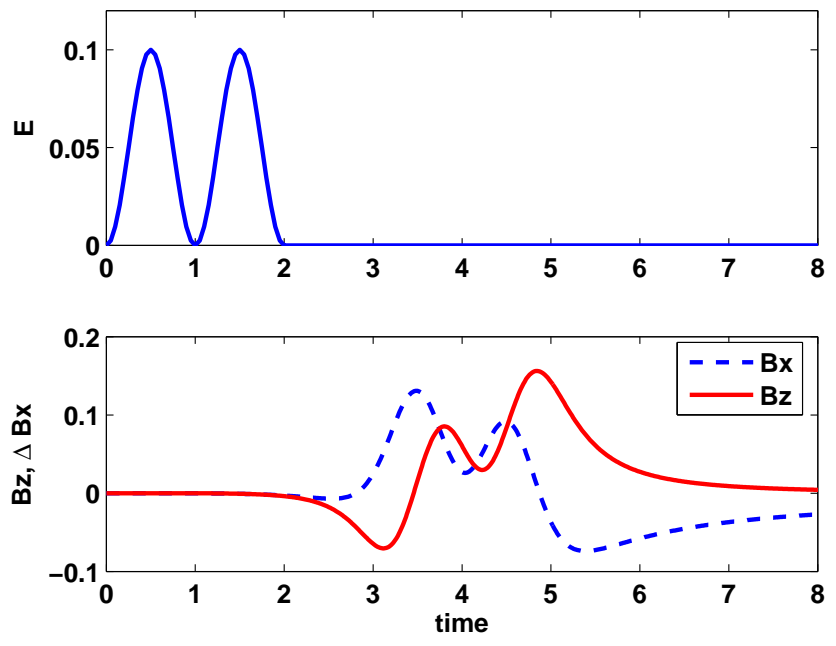

Fig. 2. Dual reconnection pulse (upper panel) and the corresponding magnetic field perturbations (lower panel).

the method of Laplace transforms, we get a simple relationship between Laplace images of the electric field, the Bzvariation, and the convolution kernel:

$E(p)=\frac{B_{z}(p)}{K_{z}(p)}$.

Once the position of the spacecraft with respect to the $\mathrm{X}$-line is known, the inverse Laplace transform of relation (2) gives $E(t)$. In reality, of course, the relative spacecraft location is not known a priori. To find it, a minimization procedure is utilized as follows: A trial spacecraft position $(\tilde{x}, \tilde{z})$ is assumed and the corresponding reconnection electric field $\tilde{E}(t)$ is obtained. Since the trial coordinates are not correct in general, the function $\tilde{E}(t)$ is usually negative on a part of the time interval. However, the real electric field must be positive. Therefore, the absolute value $|\tilde{E}(t)|$ is then inserted into the direct solution to obtain the magnetic field variations $B_{z}$, $B_{x}$. Minimizing the standard deviation between the calculated $B_{z}, B_{x}$ and those measured by the spacecraft, one can find both, the optimal electric field $E(t)$, and the position of the X-line with respect to the spacecraft.

To wind up the description of the technique, we should emphasize that our method requires magnetic data collected outside the disturbing bulge (since it exploits the inverted external solution). The method can be applied to isolated reconnection events and to composite reconnection events (consisting of several NFTE/TCR-signatures) as well.

\section{Application to THEMIS event on 22 February 2008, 04:35 UT}

We now apply the technique described above to a dual NFTE observed by THB spacecraft in the magnetotail during a substorm on 22 February 2008 around 04:35 UT. We shall not

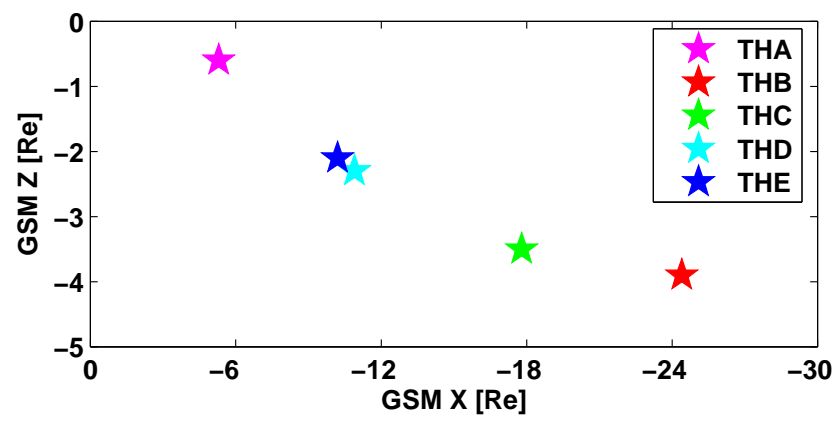

Fig. 3. Positions of THEMIS spacecraft on 22 February 2008, 04:35 UT in $(x, z)$ GSM plane.

discuss ground signatures of the substorm, noting only that they included Pi2 pulsations at Pine Ridge station, increase of THEMIS AE index to $200 \mathrm{nT}$ and the aurora intensification at $68^{\circ}$ magnetic latitude, near the location of THB, C, D, E footpoints (Liu et al., 2009). Among magnetotail aspects of the substorm we concentrate on those, which are relevant to our purpose. For further details the reader is referred to Liu et al. (2009).

At the time of interest THEMIS spacecraft were located as shown in Fig. 3, covering the region from 6 to $24 R_{E}$ in the tail. In accordance with a value of the observed $B_{x}-$ component of the background magnetic field, probes THB and THC were flying through the southern periphery of the plasma sheet $\left(B_{x}^{B} \sim-19 \mathrm{nT}, B_{x}^{C} \sim-22 \mathrm{nT}\right)$, whereas THD and THE were situated deep inside the plasma sheet, slightly to the north from the neutral sheet $\left(B_{x}^{D} \sim+2 \mathrm{nT}, B_{x}^{E} \sim\right.$ $+5 \mathrm{nT})$. All the probes detected substorm-related plasma sheet activity around 04:35 UT.

At 04:35:16 UT the probe THB detected commencement of a dual bipolar structure with the main negative pulse in $B_{z}$ preceded by the smaller positive pulse (Fig. 4). One can see that (1) change of sign of the $B_{z}$-variation approximately coincides with the extremum of $B_{x}$-variation, and (2) $v_{z}$-component of the ion velocity anticorrelates with $B_{z^{-}}$ component of the magnetic field, changing from outward flow (away from the current sheet) to inward one (toward the current sheet). These properties are typical for NFTEs (Sergeev et al., 1992), which are believed to be associated with reconnection in the tail. The polarity of $B_{z}$-variation (first slightly positive, then strongly negative) gives an evidence that the outflow bulge responsible for these remote NFTE-signatures was propagating tailward. Thus, interpreting THB observations in favour of reconnection we may conclude that the X-line was located Earthward of THB.

To our opinion, there is an ambiguity in the interpretation of THC observations (Fig. 5). Owing to indistinct character of magnetic field and velocity variations at THC, it is not clear whether the probe observed a bipolar $B_{z^{-}}$ variation of $-/+$ polarity (stating at 04:33:45 UT) or positive 


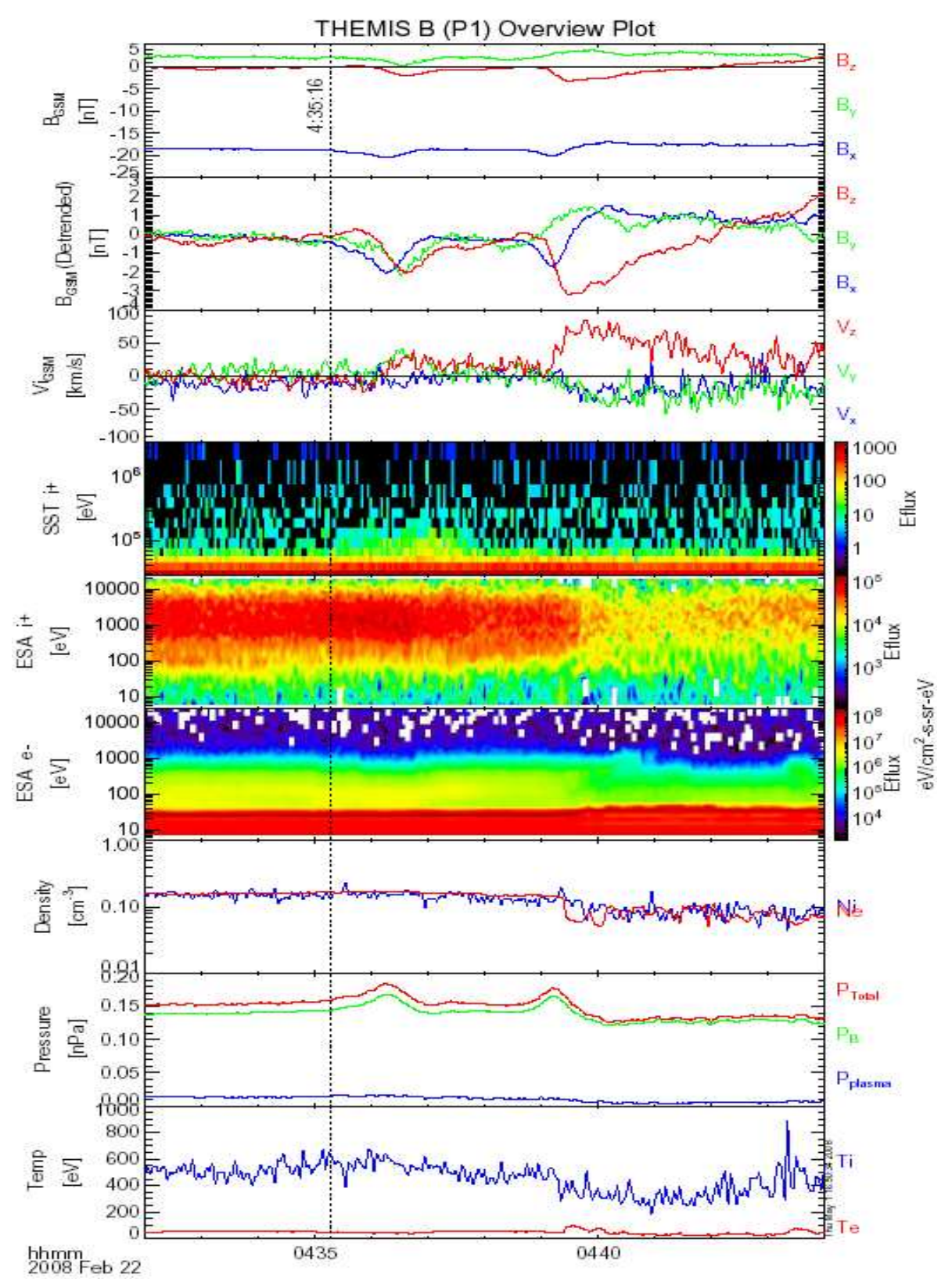

Fig. 4. Observations of THB on 22 February 2008 around 04:35 UT.

$B_{z}$-deflection (stating from 04:34:20 UT)? In the first case observations would be interpreted as the passage of a totally formed Earthward propagating bulge, which NFTEproperties (phase shift between $B_{z}$ and $B_{x}$, correlation between $B_{z}$ and $v_{z}$ ) are not well-pronounced. In the second case the data from THC may be interpreted as the onset of reconnection inflow towards the neutral sheet (indeed, the positive $B_{z}$-deflection is accompanied by the northward plasma flow), meaning that reconnection activates in the vicinity of THC and proceeds through the first stage, when the bulges are still in formation. In both cases the X-line should be lo- cated tailward of the probe THC and, possibly, very close to it (if the second interpretation is right).

Two neighbouring probes THD/THE detected similar signatures: transient dipolarization at 04:36:50 UT (abrupt increase of the $B z$-component) and Earthward flow up to $600 \mathrm{~km} / \mathrm{s}$ at the maximum (Figs. 6 and 7). An interesting thing is that dipolarization and plasma flow are not synchronized: the flow onset preceded dipolarization by $\sim 50 \mathrm{~s}$ at THD and by $\sim 30 \mathrm{~s}$ at THE. And here a question arises: what has to be considered as manifestation of arrival of the bulge at the point of THD/THE observations? The onset of ion 


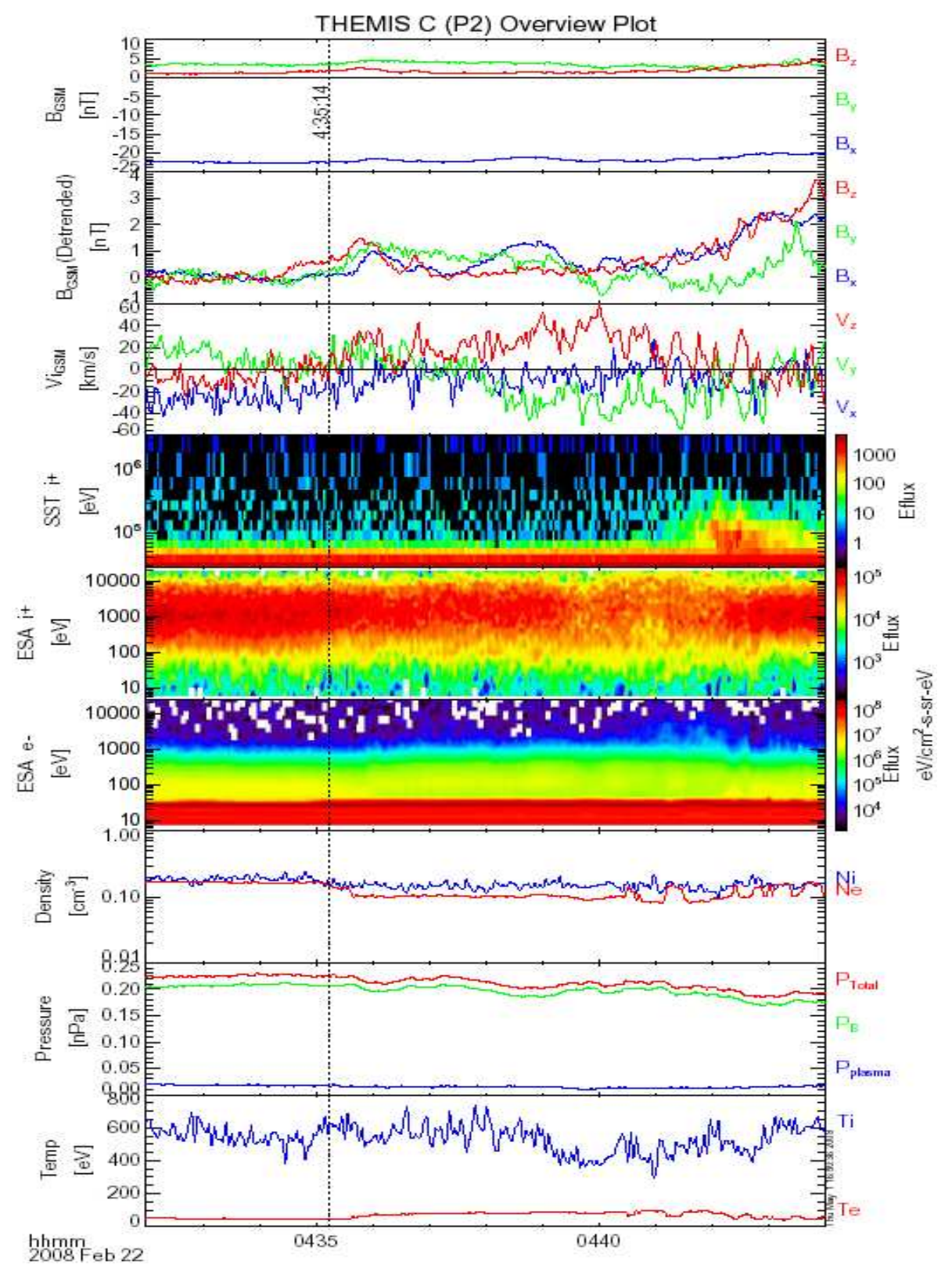

Fig. 5. Observations of THC on 22 February 2008 around 04:35 UT.

flow, accompanied by the gradual pressure/density increase, or the dipolarization onset? Commonly dipolarization is interpreted as arrival of the reconnected flux. Following this interpretation, the bulge, which carries the reconnected flux, arrived at THD/THE location at 04:36:50 UT. The earlier enhancement of the flow and the pressure/density is likely associated with the pushing effect produced by the coming bulge. It is important to note that the ion flow speed observed simultaneously with the onset of transient dipolarization was $\sim 300 \mathrm{~km} / \mathrm{s}$ on both spacecraft.
Since we do not know unambiguously the moment when THC captured the reconnection signal, we can not estimate definitely the propagation velocity of the disturbing bulge: the delay $\Delta t=185 \mathrm{~s}(04: 36: 50-04: 33: 45)$ and the distance $R=6.9 R_{E}$ between THC $\left(x_{C}=-17.8 R_{E}\right)$ and THD $\left(x_{D}=-10.9 R_{E}\right)$ give the speed of Earthward propagation $v \sim 240 \mathrm{~km} / \mathrm{s}$; the delay $\Delta t=150 \mathrm{~s}(04: 36: 50-04: 34: 20)$ corresponds to the speed $v \sim 290 \mathrm{~km} / \mathrm{s}$. The latter value is congruent with the plasma flow $(\sim 300 \mathrm{~km} / \mathrm{s})$ detected at the dipolarization front and, therefore, it is used as a preferable normalization unit to convert reconstruction results to 


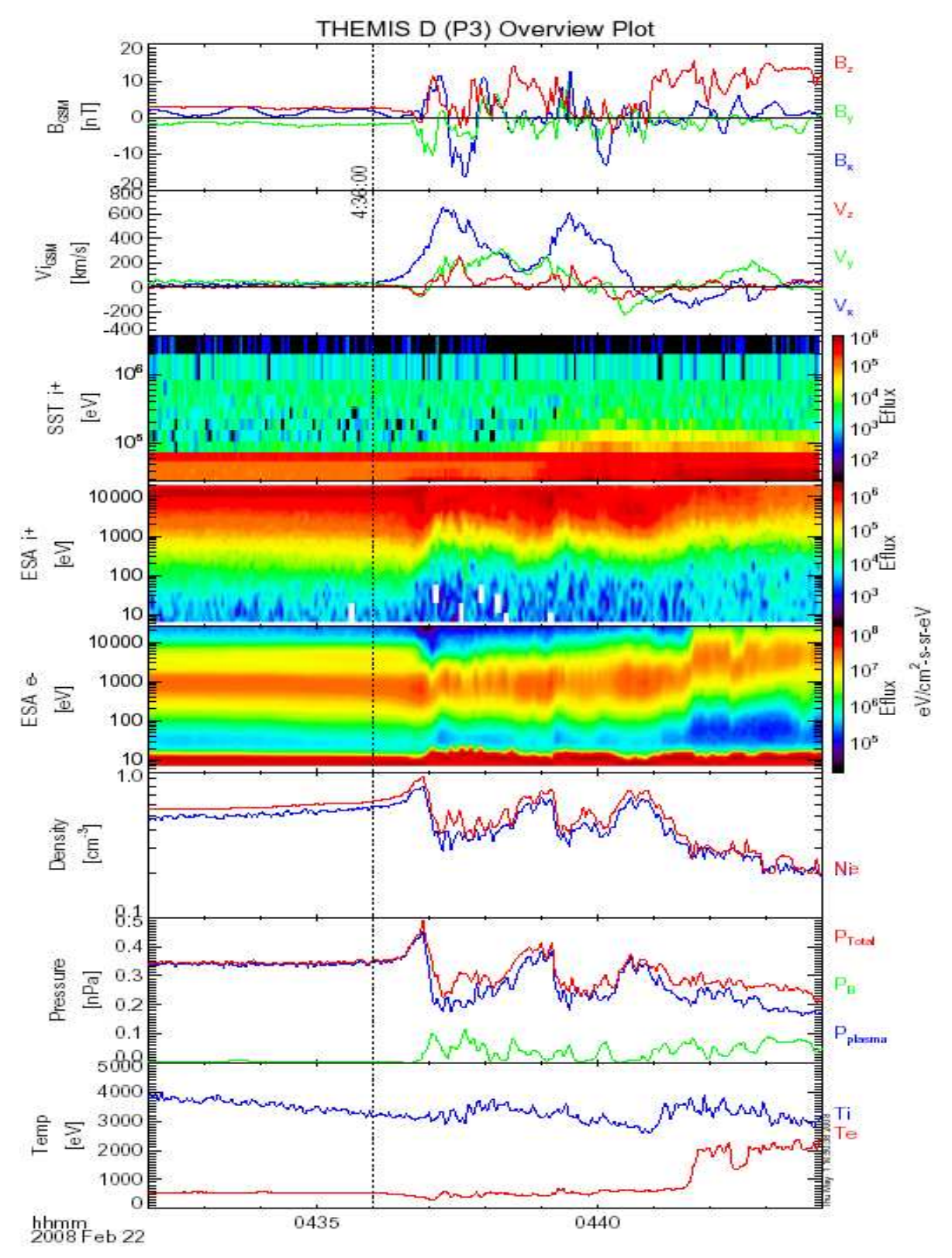

Fig. 6. Observations of THD on 22 February 2008 around 04:35 UT.

dimensional form. If we assume for a while that arrival of the bulge at THD probe is associated with the onset of ion flow at 04:36:00 UT, then we get faster propagation of the reconnection signal: $v \sim 330 \mathrm{~km} / \mathrm{s}$ for the delay $\Delta t=135 \mathrm{~s}$ (04:36:00-04:33:45) and $v \sim 440 \mathrm{~km} / \mathrm{s}$ for the delay $\Delta t=$ 100 s (04:36:00-04:34:20).

Normalization of THB magnetic data was done with respect to $B_{0}=19 \mathrm{nT}$ (background magnetic field at THB) and $T_{0}=60 \mathrm{~s}$ (typical duration of reconnection pulse). Three different variants of reconstruction have been tried: (1) using only first pulse (04:35:16-04:38:00 UT), (2) only sec- ond pulse (04:38:00-04:42:00 UT), and (3) using both pulses as one input (04:35:16-04:42:00 UT). Minimization of the standard deviation St $D$ between model magnetic perturbations and variations measured by THB spacecraft exhibited existence of a well-pronounced minimum (Fig. 8), located at $\sim-18.5 R_{E}$ in the tail. For all three variants of reconstruction the recovered $\mathrm{X}$-line position lies in the region between -18 and $-20 R_{E}$. The differences in the obtained values do not exceed the accuracy of the method, confirming that both parts of the dual bipolar structure have the same spatial origin and may be treated as one composite input. 


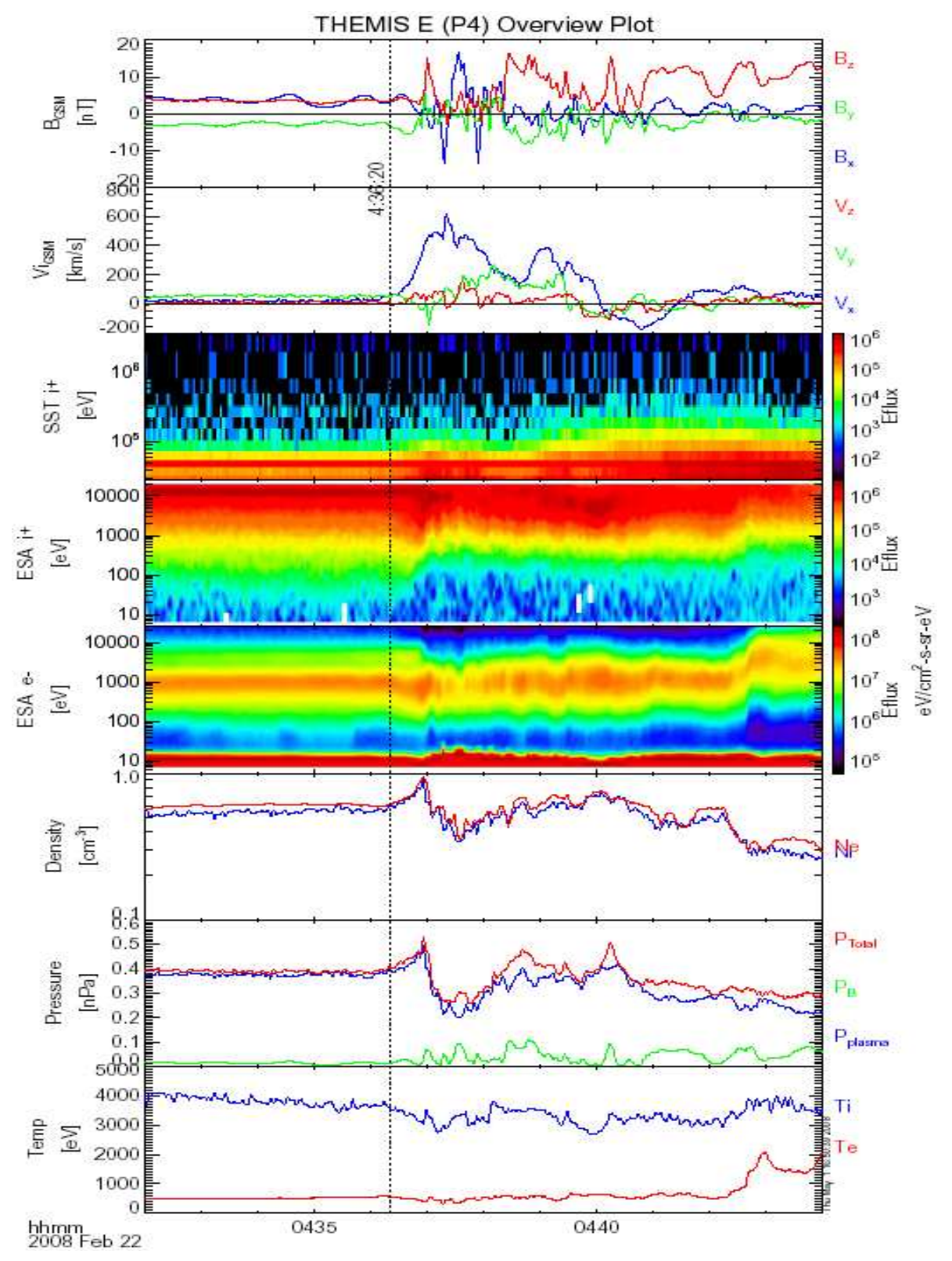

Fig. 7. Observations of THE on 22 February 2008 around 04:35 UT.

The recovered electric field consists of two successive pulses with total duration of $\sim 6 \mathrm{~min}$. The amplitude of the first pulse is $\sim 0.65 \mathrm{mV} / \mathrm{m}$, of the second one $\sim 1.1 \mathrm{mV} / \mathrm{m}$ (Fig. 9). These dimensional results were obtained with the velocity normalization unit $v=290 \mathrm{~km} / \mathrm{s}$. Use of smaller propagation velocity $v=240 \mathrm{~km} / \mathrm{s}$ reduces electric field amplitude for $\sim 0.15 \mathrm{mV} / \mathrm{m}$ and shifts the X-line tailward for $\sim 1 R_{E}$. On the contrary, use of greater values for propagation velocity $v=330 \mathrm{~km} / \mathrm{s}$ and $v=440 \mathrm{~km} / \mathrm{s}$ shifts the X-line towards the Earth to positions $\sim-17.8 R_{E}$ and $\sim-15.7 R_{E}$, correspondingly. The last position is unrealistic, since it is situated Earthward of THC probe.
The reconstructed X-line location $x_{X} \sim-18.5 R_{E}$ is in a good agreement with a simple timing, based on the capture time and positions of THB $\left(t_{B}, x_{B}\right)$ and $\operatorname{THD}\left(t_{D}, x_{D}\right)$ :

$\left(x_{D}-x_{X}\right)-\left(x_{X}-x_{B}\right)=v\left(t_{D}-t_{B}\right)$.

Varying the propagation velocity of the bulge from 200 to $1000 \mathrm{~km} / \mathrm{s}$ and assuming $10 \mathrm{~s}$ error for capture times $t_{B}$ and $t_{D}$, we get the dependence $x_{X}(v)$ shown in Fig. 10. One can see that the argument $v \sim 300 \mathrm{~km} / \mathrm{s}$ corresponds to the reconnection site at $x_{X} \sim-18.5 R_{E}$. 


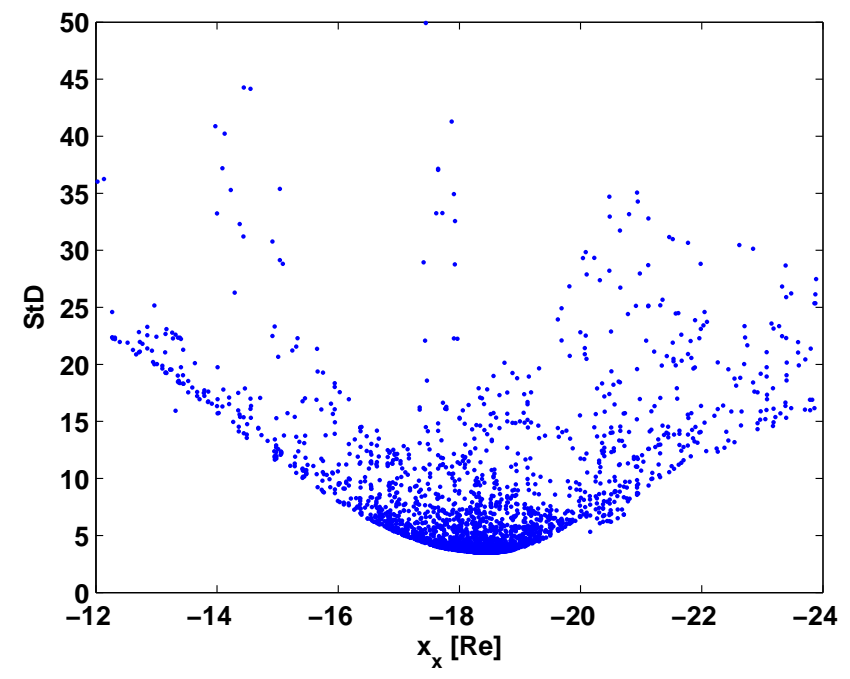

Fig. 8. Distribution of the standard deviation St D as function of the $\mathrm{X}$-line location for reconstruction variant (1).

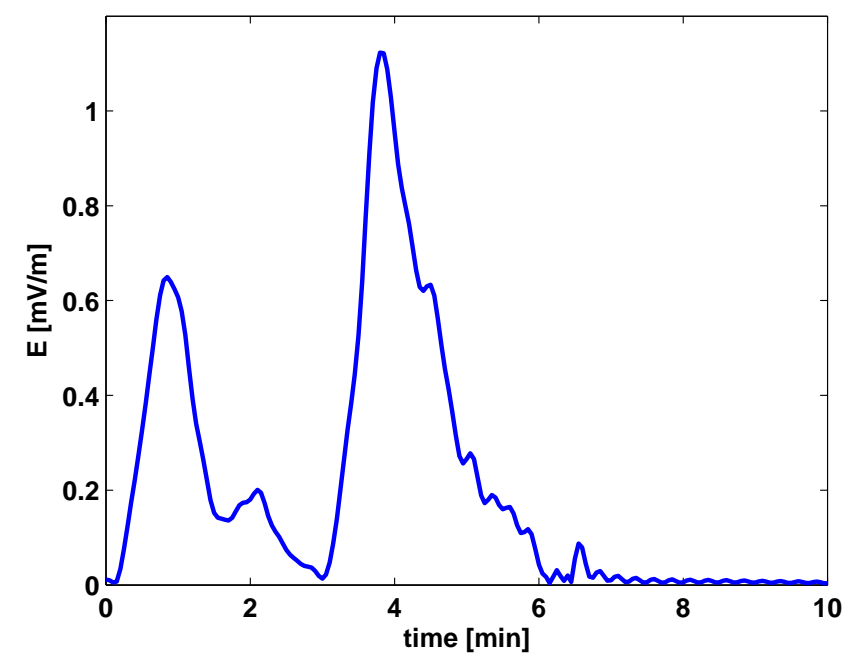

Fig. 9. Reconstructed electric field $E(t)$.

\section{Conclusions}

In the present paper we applied for the first time the inverse reconstruction technique based on the time-dependent Petschek-type reconnection model to THEMIS data. Being inspired (together with the majority of magnetosphere researchers) by capability of THEMIS mission, we hoped to trace the reconnection-associated structure through the sequence of THEMIS spacecraft and thus to get a reliable estimate of its average propagation velocity. However, we found that essential uncertainties in determination of reconnection signal commencement exist and the obtained estimate for the average propagation velocity of magnetic structure is not as reliable as it was expected.

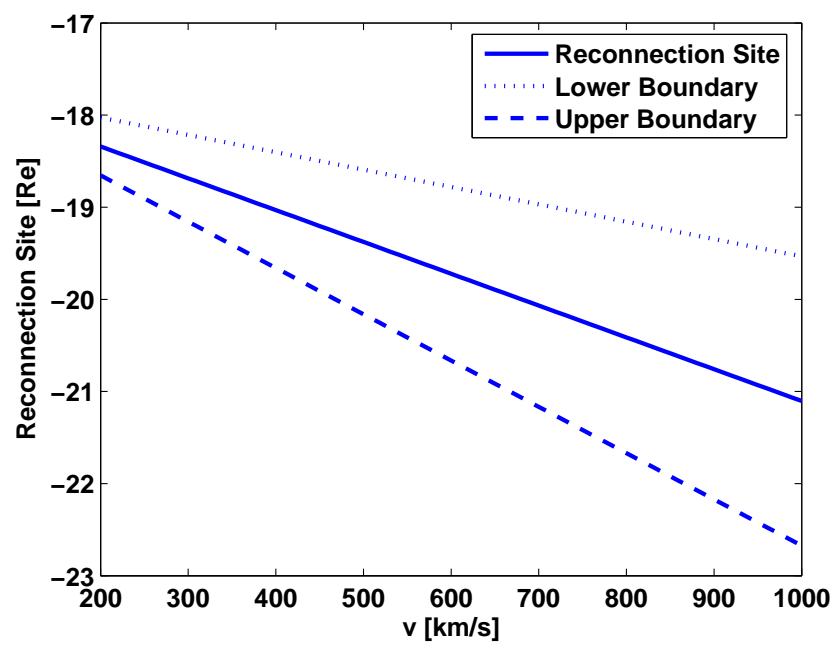

Fig. 10. Dependence of X-line location on propagation velocity.

One source of uncertainties is the difficulty to distinguish the start of the event on THD/THE probes. The simplest timing can be done by taking the first signature, that is the onset of ion flow, accompanied by the gradual pressure/density increase ahead of the NFTE-bulge. However, this timing seems to be not appropriate for our purpose: we need the arrival time of the bulge itself. Thus, we prefer to consider that it is dipolarization onset that manifests the arrival of the NFTEbulge.

Another source of ambiguity is indistinct character of the magnetic field and velocity variations at THC spacecraft (probably, caused by close location of the X-line). We have considered different timing possibilities and, in spite of the indicated uncertainties, we have got an estimate of the Xline position and the reconnection electric field which look reasonable.

Acknowledgements. This work is supported by RFBR grant No. 07-05-00776a, by RFBR/CRDF grant No. 07-05-91109, and by the Austrian "Fonds zur Förderung der wissenschaftlichen Forschung" under project P20341-N16. Also acknowledged is support by the Austrian Academy of Sciences, "Verwaltungsstelle für Auslandsbeziehungen".

Topical Editor I. A. Daglis thanks S. Dyadechkin and another anonymous referee for their help in evaluating this paper.

\section{References}

Richardson, I. G. and Cowley, S. W. H.: Plasmoid-associated energetic ion bursts in the deep geomagnetic tail: properties of the boundary layer, J. Geophys. Res., 90(A12), 12133-12158, 1985.

Richardson, I. G. and Cowley, S. W. H.: Plasmoid-associated energetic ion bursts in the deep geomagnetic tail: properties of plasmoids and the postplasmoid plasma sheet, J. Geophys. Res., 92(A9), 9997-10013, 1987. 
Biernat, H. K., Heyn, M. F., and Semenov, V. S.: Unsteady Petschek reconnection, J. Geophys. Res., 92, 3392-3396, 1987.

Heyn, M. F., Biernat, H. K., Rijnbeek, R. P., and Semenov, V. S.: The structure of reconnection layers, J. Plasma Physics, 40, 235252, 1988.

Heyn, M. F. and Semenov, V. S.: Rapid reconnection in compressible plasma, Phys. Plasmas, 3, 2725-2741, 1996.

Ivanova, V. V., Semenov, V. S., Penz, T., Ivanov, I. B., Sergeev, V. A., Heyn, M. F., Farrugia, C. J., Biernat, H. K., Nakamura, R., and Baumjohann, W.: Reconstruction of the reconnection rate from Cluster measurements: Method improvements, J. Geophys. Res., 112, A10226, doi:10.1029/2006JA012183, 2007.

Ivanova, V. V., Semenov, V. S., Ivanov, I. B., Biernat, H. K., and Kiehas, S. A.: Reconstruction of time-varying reconnection rate and X-line location, Ann. Geophys., 26, 3445-3450, 2008, http://www.ann-geophys.net/26/3445/2008/.

Liu, J., Angelopoulos, V., Frey, H., McFadden, J., Larson, D., Glassmeier, K., Mende, S., Russell, C. T., Rae, I. J., Murphy, K. R., and Apatenkov, S.: THEMIS observation of a substorm event on 04:35, 22 February 2008, Ann. Geophys., 27, 18311841, 2009,

http://www.ann-geophys.net/27/1831/2009/.
Penz, T., Semenov, V. S., Ivanova, V. V., Heyn, M. F., Biernat, H. K., and Ivanov, I. B.: Green's function of compressible Petschektype magnetic reconnection, Phys. Plasmas, 13, 052108, 2006.

Petschek, H. E.: Magnetic field annihilation, in: Physics of solar flares, edited by: Hess, W. N., NASA SP 50, 1964.

Semenov, V. S., Heyn, M. F., and Kubyshkin, I. V.: Magnetic reconnection in non-stationary case, Soviet Astronomical Journal, 27, 660-665, 1983.

Semenov, V. S., Heyn, M. F., and Ivanov, I. B.: Magnetic reconnection with space and time varying reconnection rates in a compressible plasmas, Phys. Plasmas, 11, 62-70, 2004.

Semenov, V. S., Penz, T., Ivanova, V. V., Sergeev, V. A., Biernat, H. K., Nakamura, R., Heyn, M. F., Kubyshkin, I. V., and Ivanov, I. B.: Reconstruction of the reconnection rate from Cluster measurements: first results, J. Geophys. Res., 110, A11217, doi:10.1029/2005JA011181, 2005.

Sergeev, V. A., Elphic, R. C., Mozer, F. S., Saint-Marc, A., and Sauvaud, J. A.: A two-satellite study of nightside flux transfer events in the plasma sheet, Planet. Space Sci., 40, 1551-1572, 1992. 